\title{
Quick asymptotic upper bounds for lattice kissing numbers
}

\author{
Nils-Peter Skoruppa \\ Preprint. Id: kiss.tex,v 1.4 2003/03/07 14:47:33 skoruppa Exp skoruppa
}

\begin{abstract}
General upper bounds for lattice kissing numbers are derived using Hurwitz zeta functions and new inequalities for Mellin transforms.
\end{abstract}

\section{$1 \quad$ Statement of results}

Let $\tau_{n}$ be the kissing number in dimension $n$, i.e. the maximal number of balls of equal size in Euclidean space of dimension $n$ which can touch another one of the same radius without any two overlapping. Similarly let $\lambda_{n}$ be the maximal lattice kissing number in dimension $n$, which is defined like $\tau_{n}$, but with the restriction that all balls are centred at the points of a lattice and that the centres of the kissing balls have minimal distance to the centre of the kissed one. Alternatively, $\lambda_{n}$ is the maximal number of minimal vectors which a lattice $L$ in $\mathbb{R}^{n}$ can have. The precise values of $\lambda_{n}$ and $\tau_{n}$ are known only for finitely many values of $n[\mathrm{C}-\mathrm{S}]$. Note that $\lambda_{n} \leq \tau_{n}$. The first time when this inequality is strict occurs for $n=9$. Concerning the asymptotic behaviour of $\tau_{n}$ one knows $\tau_{n} \geq(1.15470 \ldots)^{n(1+0(1))}[\mathrm{W}]$, and one has the following asymptotic estimate to above of Kabatiansky-Levenshtein [K-L]

$$
\tau_{n} \leq 2^{0.401 n(1+o(1))}=(1.32042 \ldots)^{n(1+o(1))} .
$$

As general reference for these and more informations on kissing numbers we refer to $[\mathrm{C}-\mathrm{S}]$ or $[\mathrm{Z}]$.

In the present note we shall prove a general upper bound for $\lambda_{n}$, which we now describe. Assume

1. $f: \mathbb{R}_{\geq 0} \rightarrow \mathbb{R}_{\geq 0}$ is a nonzero decreasing and continuous function.

\footnotetext{
${ }^{0} 2000$ Mathematics Subject Classification 11H31 11M35 52C17
} 
2. $f(t)$ and the function $F(t)$ defined by $F(|y|):=\int_{\mathbb{R}^{n}} f(|x|) \mathrm{e}^{-2 \pi i x y} d x$ are $O\left(t^{-(n+\varepsilon)}\right)$ for some $\varepsilon>0$ as $t$ tends to infinity. Here $x y$ denotes the usual scalar product on $\mathbb{R}^{n}$ and $|x|=\sqrt{x x}$.

3. The Mellin transform $\gamma(s)=\int_{0}^{\infty} F\left(t^{1 / n}\right) t^{s} \frac{d t}{t}$, can be continued to a holomorphic function in a vertical strip which contains a real number $c>1$ such that

$$
\gamma(c)=\max _{\operatorname{Re}(s)=c}|\gamma(s)|
$$

Note that the Fourier transform of $f(|x|)$ is radially symmetric, which justifies to write it in the form $F(|y|)$ with a suitable function $F(t)$ of one real variable $t \geq 0$. The function $F$ is known in the literature as Hankel transform of $f$. Note furthermore that the integral defining $\gamma(s)$ is absolutely convergent for $0<\operatorname{Re}(s)<1+\frac{\varepsilon}{n}$. Finally note that any measurable function $f$ satisfying 2 . is automatically continuous.

Theorem 1. Under the above hypothesis 1.-3. one has for $\lambda_{n}$, the maximal lattice kissing number of dimension $n$, the upper bound

$$
\lambda_{n} \leq \frac{2 F(0)}{c(2 c-1) \gamma(c)} \exp \left(1+\frac{2 c}{c-1}+c \frac{\gamma^{\prime}}{\gamma}(c)\right)
$$

Supplement to Theorem 1. If, in addition, $F$ is nonnegative then, for all $c>1$ where the Mellin transform of $F$ converges, one has

$$
\lambda_{n} \leq \frac{F(0)}{c(c-1) \gamma(c)} \exp \left(1+\frac{c}{c-1}+c \frac{\gamma^{\prime}}{\gamma}(c)\right) .
$$

Note that, for nonnegative $F$, the condition 3 . is automatically satisfied with every $c$ as in the supplement.

The first upper bound is worse than the second one by a factor strictly larger than $\mathrm{e}=\exp (1)$ and tending to e if $c$ tends to infinity. However, the first bound applies to a larger variety of functions $f$ than the second one since in Theorem 1 the Hankel transform $F$ is not required to be nonnegative.

The simplest function satisfying the hypothesis $1 .-3$. is certainly $f(t)=$ $\exp \left(-t^{2}\right)$. Here the supplement gives the bound $1.64^{n(1+o(1))}$, which is far off the Kabatiansky-Levenshtein bound. One can do better by using instead $f(t)=\max \left(0,1-t^{2}\right)^{p}$ for $p \gg 0$. In this case, however, we cannot apply the bound of the supplement since the corresponding functions $F$ are not nonnegative; but condition 3. is still satisfied (cf. section 3). By applying Theorem 1 to these functions one obtains

Theorem 2. $\lambda_{n} \leq 1.3592^{n(1+o(1))}$. 
This is still worse than the Kabatiansky-Levenshtein bound. However, the methods used in this article are quite simple compared to the more subtle arguments in $\mathrm{K}-\mathrm{L}$. Moreover, we do not know whether it is not possible to make still a better choice for $f$ for improving the asymptotic estimate. Whereas the asymptotic bound of Theorem 2 is not too bad, the bounds for specific dimensions using the functions $f(t)=\max \left(0,1-t^{2}\right)^{p}$ are quite poor: $\lambda_{2} \leq 12, \lambda_{3} \leq 31$, etc.. Again it is possible that more suitable choices of $f$ for particular dimensions may yield better results.

In the next section we shall prove Theorem 1 and its supplement, and in section 3 we shall give the details for deducing Theorem 2 from Theorem 1 . Parts of this work use ideas already presented in [F-S] and in [S].

\section{Proof of Theorem 1}

Proof. For bounding $\lambda_{n}$ to above we may and will restrict to lattices $L$ in $\mathbb{R}^{n}$ with minimal length equal to 1 . Thus we have to show that, for any such $L$, the number $a_{1}(L)$ of points in $L$ with distance 1 to the origin is bounded above by the right hand side of the inequality of Theorem 1 . We shall actually show that the Hurwitz zeta function

$$
D(s)=\sum_{x \in L \backslash\{0\}}|x|^{-n s}
$$

at $s=c$ (where the sum defining $D(s)$ converges absolutely since $c>1$ ) is bounded to above by the right hand side. Since trivially

$$
a_{1}(L) \leq D(c)
$$

the estimate for $D(c)$ then proves the theorem.

Under the hypothesis 1 and 2 the Poisson summation formula is valid, i.e.

$$
t \sum_{x \in L} F\left(t^{1 / n}|x|\right)=\mathrm{g}(L)^{1 / 2} \sum_{x \in L^{*}} f\left(t^{-1 / n}|x|\right),
$$

where both sums are absolutely convergent [S-W], p. 252 (VII:Corollary 2.6). Here $L^{*}$ denote the dual lattice of $L$ (i.e. the set of all $y \in \mathbb{R}^{n}$ such that $y x \in \mathbb{Z}$ for all $x \in L$ ), and $\mathrm{g}(L)$ the Gram matrix of any $\mathbb{Z}$-basis of $L$.

Denote by $\theta(t)$ the sum on the left with the term $F(0)$ omitted. Thus the left hand side equals $t(F(0)+\theta(t))$. This is an increasing real valued function of $t$, since $f\left(t^{-1}\right)$ (and hence the right hand side) is real valued and increasing by assumption 1 . 
For the Mellin transform $\Lambda(s)$ of $\theta(t)$ we find

$$
\Lambda(s)=\int_{0}^{\infty} \theta(t) t^{s} \frac{d t}{t}=\gamma(s) \sum_{x \in L \backslash\{0\}}|x|^{-n s}=\gamma(s) D(s) .
$$

The Mellin transform is absolutely convergent for

$$
1<\operatorname{Re}(s)<1+\frac{\varepsilon}{n},
$$

and in this domain interchanging the integral and the sum over $x$ is indeed justified, as is easily deduced from the fact that the Mellin transform of $F\left(t^{1 / n}\right)$ is absolutely convergent for $0<\operatorname{Re}(s)<1+\varepsilon / n$ (by hypothesis 2 .), and that the Hurwitz zeta function of $L$ converges for $\operatorname{Re}(s)>1$.

Before giving the rest of the proof, we show how to deduce our estimate using a short argument which, however, is based on assumptions not holding true in general, but has the advantage of being rapid and suggestive. Assume that $\Lambda(s)$ can be analytically continued to a strip containing 0 and $c$, with poles only at 0 and 1 , which are simple, and with residue $-F(0)$ at $s=0$. This is a standard situation, which holds true for instance with $f(t)=\exp \left(-t^{2}\right)$. Then we may consider the function $g(s)=s(s-1) \Lambda(s)$. Note that $g(0)=F(0)$. Assume further that $\log g(s)$ is defined in $[0, c]$ and convex on this interval. Then

$$
\log g(c)-\log g(0) \leq c(\log g)^{\prime}(c)=c \frac{g^{\prime}}{g}(c) .
$$

Taking exponentials in this inequality gives

$$
D(c) \leq \frac{F(0)}{c(c-1) \gamma(c)} \exp \left(1+\frac{c}{c-1}+c \frac{\gamma^{\prime}}{\gamma}(c)+c \frac{D^{\prime}}{D}(c)\right) .
$$

Dropping the term containing $D^{\prime}$ (which is negative since $D(s)$ is decreasing for $s>1$ ) we recognise the estimate for $D(c)$ as in the supplement of Theorem 1.

The mentioned assumption about $\Lambda(s)$ will in general be false. Nevertheless, if $F$ is nonnegative, the inequality for $g(s)$ holds still true for every $c>1$ where the Mellin transform of $F$ converges. For the proof we rewrite it as

$$
F(0) \geq g(c) \exp \left(-c \frac{g^{\prime}}{g}(c)\right)
$$

This inequality is equivalent to the statement that for all $a>0$ one has

$$
F(0) \geq\left(g(c)-c g^{\prime}(c)+c g(c) \log a\right) a^{-c}=-c^{2} \frac{d}{d s}\left[\frac{a^{-s}}{s} g(s)\right]_{s=c} .
$$


Indeed, taking $\log a=\frac{g^{\prime}}{g}(c)$ gives the first inequality, and for this $a$ the right hand side of the second one attains its maximum. For proving the latter estimate we consider, for fixed $s>1$ (where $\gamma(s)$ converges), the function

$I(a)=a F(0) / s^{2}-a \int_{0}^{a} \theta(t) \log (t / a)(t / a)^{s} \frac{d t}{t}=\int_{0}^{\infty}(F(0)+\theta(a t)) a t w(t) \frac{d t}{t}$,

where $w(t)=-t^{s-1} \log \max (1, t)$. The second identity is easily justified by the substitution $t \leftarrow a t$. Since $w(t)$ is nonnegative and $F(0)+\theta(t) t$ is increasing, the function $I(a)$ is an increasing function. Hence $I^{\prime}(a) \geq 0$. Using the first formula for $I(a)$ for computing the derivative we thus obtain

$F(0) / s^{2} \geq-\int_{0}^{a} \theta(t)(1+(s-1) \log (t / a))(t / a)^{s} \frac{d t}{t}=-\frac{d}{d s} \frac{a^{-s}}{s} \int_{0}^{a} \theta(t) t^{s} \frac{d t}{t}$.

Since $F(t)$ and hence $\theta(t)$ is nonnegative this inequality remains valid if we replace $\int_{0}^{a}$ by $\int_{0}^{\infty}$. Writing $c$ for $s$ this is then the desired inequality, which proves the supplement to Theorem 1.

In the general case, i.e. for not necessarily nonnegative $F$, we shall be able to prove an estimate similar to the one above, however with a factor $1 / 2$ and a slightly different $g$. Namely, we shall prove

$$
F(0) \geq \frac{1}{2} g(c) \exp \left(-c \frac{g^{\prime}}{g}(c)\right),
$$

where

$$
g(s)=\frac{2 c-1}{2 c-1-s} s(s-1) \Lambda(s) .
$$

From this we deduce as before an upper bound for $D(c)$ and then Theorem 1 .

As before the inequality for $F(0)$ is equivalent to the statement that for all $a>0$ one has

$$
F(0) \geq-\frac{c^{2}}{2} \frac{d}{d s}\left[\frac{a^{-s}}{s} g(s)\right]_{s=c} .
$$

For proving this inequality we set

$$
\begin{aligned}
H(a)=a F(0) / c^{2}+\frac{1}{2 \pi i} \int_{\operatorname{Re}(s)=c-\varepsilon} \frac{a^{1-s} g(s)}{s(s-1)} & \cdot \frac{d s}{(s-c)^{2}} \\
= & \int_{0}^{\infty}(F(0)+\theta(a t)) a t v(t) \frac{d t}{t},
\end{aligned}
$$

where

$$
v(t)=\frac{1}{2 \pi i} \int_{\operatorname{Re}(s)=c-\varepsilon} \frac{2 c-1}{2 c-1-s} \cdot \frac{t^{s-1} d s}{(s-c)^{2}},
$$


The second identity is easily justified by making on the right hand side the substitution $t \leftarrow t / a$, replacing $v(t)$ by its integral representation, interchanging integrals and using finally $\int_{0}^{\infty} v(t) d t=1 / c^{2}$. For $\varepsilon$ we may choose and positive real number such that $c-\varepsilon>1$ and such that the Mellin transform of $F(t)$ (and hence of $\theta(t)$ ) converges absolutely at $s=c-\varepsilon$.

By a simple calculation we see

$$
v(t)=\frac{(2 c-1)}{(c-1)^{2}} \cdot\left\{\begin{array}{ll}
t^{c-1}\left(-\log t^{c-1}+t^{c-1}-1\right) & \text { if } t<1 \\
0 & \text { if } t \geq 1
\end{array} .\right.
$$

Hence $v(t)$ is nonnegative, and since $(F(0)+\theta(t)) t$ is increasing, we see that $H(a)$ is an increasing function. Hence $H^{\prime}(a) \geq 0$, i.e.

$$
F(0) / c^{2} \geq \frac{1}{2 \pi i} \int_{\operatorname{Re}(s)=c-\varepsilon} \frac{a^{-s} g(s)}{s} \cdot \frac{d s}{(s-c)^{2}} .
$$

Now, for $s=c+i t$ with real $t$, one has

$$
\operatorname{Re} \frac{a^{-s} g(s)}{s} \leq\left|\frac{a^{-s} g(s)}{s}\right| \leq \frac{g(c) a^{-c}}{c}
$$

as follows easily from the assumption that $|\gamma(s)| \leq \gamma(c)$ for $s=c+i t$. We may hence apply the following lemma to estimate the last integral to below by $-\frac{1}{2} \frac{d}{d s}\left[\frac{g(s)}{s} a^{-s}\right]_{s=c}$, which is the desired inequality. This proves the theorem.

The following lemma was proven, in a slightly different form, in [F-S].

Lemma. Let $f(s)$ be a bounded and holomorphic function in some strip $a<\operatorname{Re}(s)<b$, real valued for real $s$. Assume that for some $a<c<b$ we have

$$
\sup _{t \in \mathbb{R}} \operatorname{Re} f(c+i t)=f(c) .
$$

Then, for all $\varepsilon>0$ with $a<c-\varepsilon$, one has

$$
\frac{1}{2 \pi i} \int_{\operatorname{Re}(s)=c-\varepsilon} \frac{f(s) d s}{(s-c)^{2}} \geq-\frac{1}{2} f^{\prime}(c) .
$$

Proof. Since $f(s)$ is bounded, the integrand of the integral in question is $O\left(t^{-2}\right)$ for $t=\operatorname{Re} s \rightarrow \pm \infty$. Hence we can replace the path of integration by the line segment from $c-i \infty$ to $c-i \delta$, then along the left half circle with centre $c$ to $c+i \delta$, and finally the line up to $c+i \infty$. Here $\delta$ is any positive number. For the integral along the half circle $\gamma$ we find

$$
\frac{1}{2 \pi i} \int_{\gamma}=\frac{1}{\delta} \int_{3 / 4}^{1 / 4} f\left(c+\delta \mathrm{e}^{2 \pi i t}\right) \mathrm{e}^{-2 \pi i t} d t=\frac{1}{\pi \delta} f(c)-\frac{1}{2} f^{\prime}(c)+O(\delta) .
$$


For the integrals along the line segments we find, using $\overline{f(z)}=f(\bar{z})$ (since this is true for real $s$ by assumption),

$$
\frac{1}{2 \pi i}\left(\int_{c-i \infty}^{c-i \delta}+\int_{c+i \delta}^{c+i \infty}\right)=-\frac{1}{\pi} \int_{\delta}^{\infty} \frac{\operatorname{Re} f(c+i t) d t}{t^{2}} \geq-\frac{1}{\pi \delta} f(c) .
$$

For the inequality we used $\operatorname{Re} f(c+i t) \leq f(c)$. On taking $\delta \rightarrow 0$ the lemma follows.

\section{Proof of Theorem 2}

Proof. For $p \geq 0$, the function

$$
f(t):=\max \left(0,1-t^{2}\right)^{p}
$$

is nonnegative, decreasing and continuous. We show that $f$ satisfies the assumptions of Theorem 1.

One has

$$
F(t)=C \Gamma(q) J_{q-1}(2 \pi t) /(\pi t)^{q-1} \quad\left(q=\frac{n}{2}+p+1\right)
$$

with $J_{q-1}$ being the Bessel function of order $q-1$ [S-W], p. 171 (IV:Theorem $4.15)$, and with a positive constant $C$. Note that $F(0)=C$. Using

$$
J_{q-1}(t)=O\left(t^{-1 / 2}\right)
$$

[S-W], p. 158 (IV:Lemma 3.11), we see that $F$ satisfies the hypothesis 2. of Theorem 1 as long as

$$
\left.q>n+\frac{1}{2} \quad \text { (i.e. } p>\frac{n-1}{2}\right) .
$$

In the following we assume this inequality. The Mellin transform of $F\left(t^{1 / n}\right)$ equals

$$
\gamma(s)=C \frac{n}{2} \pi^{-n s} \frac{\Gamma(q) \Gamma\left(\frac{n s}{2}\right)}{\Gamma\left(q-\frac{n s}{2}\right)}
$$

([S-W], p. 174 or Titchmarch) The hypothesis 3. is therefore satisfied for $c=q / n$ since, for any $s$ with real part $c$, one has $|\gamma(s)|=\gamma(c)$.

Thus, by Theorem 1, we obtain the estimate

$$
\lambda_{n} \leq \frac{4 n}{q(2 q-n) \Gamma(q)} \exp \left(1+\frac{2 q}{q-n}+q \psi\left(\frac{q}{2}\right)\right) .
$$


Using

$$
\log \Gamma(q)=q \log q-q+o(q), \quad \psi(q)=\log q+O\left(q^{-1}\right) \quad(q \rightarrow \infty),
$$

we obtain, for all fixed small $10^{-4} \geq \varepsilon>0$, by setting $q=(1+\varepsilon) n$, the estimate

$$
\lambda_{n} \leq \exp (n(1+\varepsilon)(1-\log 2)+o(n)) \leq 1.3592^{n(1+o(1))}
$$

as $n \rightarrow \infty$. This proves Theorem 2 .

\section{References}

[C-S] J.H. Conway and N.J.A. Sloane, Sphere packings, lattices and groups. Springer Verlag, 1988.

[F-S] E. Friedman and N.-P. Skoruppa, Lower Bounds for the $L^{p}$-norm in terms of the Mellin transform, Bull. London Math. Soc. 25 (1993), $567-572$.

[K-L] G.A. Kabatiansky and V.I. Levenshtein, Bounds for packings on a sphere and in space, Problems of Information Transmission 14 (No. 1, 1978) $1-17$

[S] N.-P. Skoruppa, Quick lower bounds for regulators of number fields, Enseign. Math. t. 39 (1993), 137-141.

[S-W] E. Stein and G. Weiss, Introduction to Fourier analysis on Euclidean spaces. Princeton University Press 1971.

[W] A.D. Wyner, Capabilities of bounded discrepancy decoding, AT\&T Technical Journal 44 (1965), 1061-1122.

[Z] C. Zong, Sphere Packings. Springer Verlag, 1999.

Nils-Peter Skoruppa

Fachbereich Mathematik, Universität Siegen

Walter-Flex-Straße 3, 57068 Siegen, Germany

http://www.countnumber.de

skoruppa@math.uni-siegen.de 